\title{
LEVANTAMENTO DE CARACTERISTICAS EPIDEMIOLÓGICAS DO SARAMPO EM SÃO JOSÉ DO RIO PRETO (BRASIL) - 1973
}

Eduardo Paulo BOSKOVITZ *

Cristina Helena BENETTI *

Rackel Ivannie Moreira de TOLEDO*

RSPU-B/215

Boskovitz, E. P. et al. - Levantamento de características epidemiologicas do sarampo em São José do Rio Prêto (Brasil) - 1973. Rev. Saúde públ., S. Paulo, 8:181-6, 1974.

RESUMo: Considerando-se o sarampo como infecção com grande importância médico-sanitária atual, estudou-se sua incidência numa cohorte de 5 anos (entre 2 - 7 anos atuais) em região urbana de São José do Rio Prêto (Brasil) evidenciando-se a elevação da incidência progressivamente em idades mais jovens. Utilizando-se de método de investigação acessivel em noso meio - entrevistas - determinou-se a ação profilática da vacina específica (96\%) e a demanda hospitalar devido ao sarampo (758 leitos/dia) na área estudada nos últimos 5 anos.

UNITERMos: Sarampo*; Epidemiologia *; Morbidade*; Profilaxia vacinal; Demanda Hospitalar.

\section{INTRODUÇ $\mathrm{A}$}

Estudos epidemiológicos realizados nos EUA $^{1}$ e Checoslováquia ${ }^{6}$ têm realçado a importância médica, social e sanitária do sarampo. Entre nós, conforme últimos estudos no levantamento de mortalidade na infância ${ }^{5}$, tem sido evidenciada esta virose como problema de absoluta prioridade, quer pelas baixas condições sócioeconômicas de grande contingente de população infantil acarretando elevados índices de mortalidade, quer pela eficácia já amplamente demonstrada dos meios de imunização existentes na prevenção do acometimento desta doença exantemática.

Procuramos, assim, na área em que tra- balhamos, conhecer alguns dos parâmetros da incidência do sarampo, tendo vários objetivos em mente:

a) aplicação prática da metodologia epidemiológica em atividades de campo com alunos da Faculdade Regional de Medicina de São José do Rio Prêto (FARME) ;

b) análise dos grupos etários mais atingidos numa amostra de população infantil, em zona urbana, na vigência de vacinação anti-sarampo em nosso meio;

* Do Departamento de Medicina Preventiva da Faculdade Regional de Medicina - São José do Rio Prêto, SP - Brasil. 
BOSKOVITZ, E. P. et al. - Levantamento de caracteristicas epidemiológicas do sarampa em São José do Rio Prêto (Brasil) - 1973. Rev. Saúde públ., S. Paulo, 8:181-6, 1974.

c) avaliação inicial da gravidade da doença em meio onde a prevenção eficaz - vacina - começa a ser estendida à população;

d) etapa inicial de estudos que possibilite medir, numa $2 .^{a}$ fase, a atuação dos mecanismos de proteção nesta população (vacinação) .

\section{MATERIAL E MÉTODOS}

\subsection{Método}

\subsubsection{Amostra Populacional}

Dada a maior prevalência do sarampo no grupo etário pré-escolar e infantil, estabelecemos nossa amostra para o grupo etário que se encontra entre $2-7$ anos (nascidos entre $1 . \% 05 / 1966$ a $30 / 04 /$ 1971). Limitou-se para 5 anos o intervalo de idade, pois, com informaçöes obtidas por entrevista poder-se-ia prejudicar a confiabilidade dos dados obtidos. Foram entrevistadas mães ou responsáveis destas crianças domiciliadas na área em estudo.

\subsubsection{Area}

Utilizou-se a área de atuação do Centro de Saúde Escola que tem limites claramente determinados na área urbana de Sáo José do Rio Prêto, em bairros próximos ao Hospital e Escola de Medicina.

Nesta área sortearam-se $50 \%$ das quadras previamente numeradas, as quais tiveram suas casas recenseadas quanto à existência no domicílio:
a) de indivíduos menores de 2 anos;
b) no grupo etário $2-7$ anos; e
c) com mais de 7 anos completos.

\subsubsection{Entrevista}

Selecionadas as casas onde havia pelo menos uma criança com idade dentro do limite etário estabelecido, a mãe ou responsável era entrevistada por acadêmicos de Medicina, previamente treinados por elementos docentes do Departamento de Medicina Preventiva da FARME.

Seguia-se um roteiro onde era indagade da ocorrência ou não de sarampo, no(s) elemento(s) objeto(s) deste levantamento; em caso $(+)$, indagava-se a época em que ocorrera, quem fizera o diagnóstico, se houve ou não hospitalização e o número de dias decorridos. Finalmente, o entrevistador solicitava algum documento que provasse a idade da criança e a comprovação (caderneta de vacinação) ou não de imunização específica contra o sarampo e a data de sua aplicação.

As entrevistas obtidas eram encaminhadas a um dos quatro orientadores do trabalho (docentes) que observando alguma falha $e$ julgando haver necessidade de alguma informação complementar, devolvia-a para que fosse completada em nova visita.

\subsection{Material}

Das 632 quadras existentes foram sorteadas $316(50 \%)$. Nestas havia 2.398 casas distribuídas nos grupos etários em foco segundo Tabela 1.

TABELA- 1

Distribujção dos grupos etários nas casas da área estudada

\begin{tabular}{lc}
\hline Grudos etários & N.o de casas \\
$<2$ anos & 191 \\
$>7$ anos & 1.242 \\
sem informaçóo & 11 \\
$2-1$ anos & 954 \\
\hline Total & 2.398 \\
\hline
\end{tabular}


Boskovitz, E. P. et al. - Levantamento de caracteristicas epidemiológicas do sarampo em São José do Rio Prêto (Brasil) - 1973. Rev. Saúde públ., S. Paulo, 8:181-6, 1974.

Portanto a nossa análise se refere a 954 casos onde moram indivíduos que se encontravam entre 2 anos completos e 7 incompletos até 30/04/1973.

\section{RESULT A D O S}

\subsection{Valor Profilático da Vacina}

Analisando-se estatisticamente o grupo de crianças que teve o sarampo em relação a ter ou não recebido a vacina específica, obteve-se: $\mathrm{x}^{2}=303,1(\leq \mathrm{p} 0,01)$ (Tabela 2).

TABEL 2

Distribuição das crianças em relação ao sarampo e à vacinação especifica

\begin{tabular}{c|c|c|c}
\hline Vacinação & $\operatorname{sim}$ & Não & Total \\
\hline Sim & 21 & 573 & 594 \\
\hline Não & 305 & 338 & 643 \\
\hline Total & 326 & 911 & 1.237 \\
\hline
\end{tabular}

3.2. Dos 21 casos referidos como tendo sido vacinados e acometidos pela doença deve-se observar que:

a) $10(48 \%)$ tiveram, provavelmente, uma reação vacinal uma vez que a ocorrência de doença foi relatada num período que variou entre 15 e 20 dias após a vacinação específica. Ressalve-se a possibilidade da vacinação ter coincidido com o período de incubação da doença em si.

b) dos 11 casos restantes, 9 (43\%) foram seguramente vacinados nas campanhas multi-vacinais realizadas nos últimos 2 anos; os $2(9 \%)$ casos restantes, pela data da vacinação é pouco provável que tenham recebido a vacina adequada. Portanto $(305+$ 10) 315 é o número de crianças vacinadas e que não tiveram a doença.

Conclui-se, pois, que a efetividade da vacina teria sido:

$$
315 / 326=0,96 \text { ou seja } 96 \%
$$

3.3. Distribuição etária da doença na amostra estudada:

- Por cohortes (Figura 1);

- Por idade (Tabela 3).

Analisando-se a distribuição por cohor. tes de nascimento a partir do ano de 1966-67, observa-se na Figura 1 que a incidência da doença nos grupos etário: menores (atualmente com 2 e 3 anos) é mais elevada do que naqueles com idades maiores. Parece haver um deslocamento

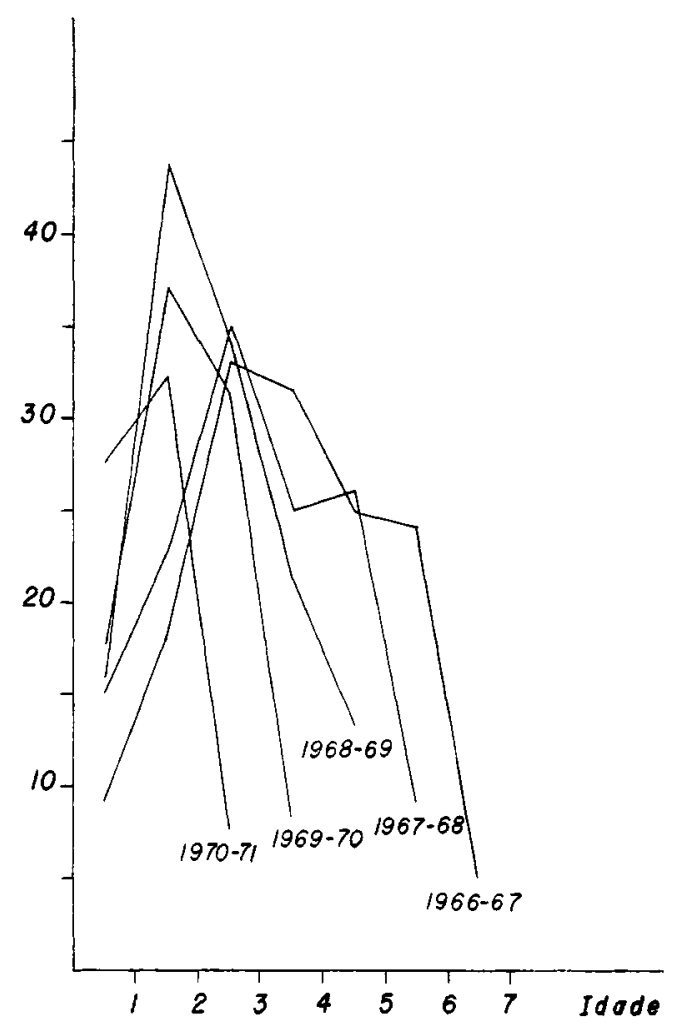


BOSKOVITZ, E. P. et al. - Levantamento de caracteristicas epidemiológicas do sarampo em São José do Rio Prêto (Brasil) - 1973. Rev. Saúde públ., S. Paulo, 8:181-6, 1974.

TABELA 3

Frequèencia do sarampo no grupo etário com 2 - 7 anos de idade - São José do Rio Prêto - 1973 - Area do CSE

Grupo etário $\mid$\begin{tabular}{c} 
f \\
\cline { 2 - 3 }
\end{tabular}

\begin{tabular}{rrr}
\hline $0-\mid 1$ & 85 & 14,9 \\
$1-\mid 2$ & 155 & 27,2 \\
$2-\mid 3$ & 140 & 24,6 \\
$3-\mid 4$ & 86 & 15,1 \\
$4-\mid 5$ & 65 & 11,4 \\
$5-\mid 6$ & 33 & 5,7 \\
$6-\mid 7$ & 5 & 0,8 \\
\hline Total & 569 & 99,7 \\
\hline
\end{tabular}

Obs.: Das 594 crianças acometidas pelo sarampo, de 25 não se obteve a informação da época em que ocorreu o sarampo.

da incidência, nestes últimos 5 anos, para as idades mais jovens.

Poder-se-ia explicar o fato pela maior concentração de população na área em apreço ou, talvez, por estarmos em anos nos quais há a elevação cíclica da incidência desta virose exatamente pelo aumento da concentração dos suscetiveis.

3.4. Na Tabela 3 evidencia-se, igualmente, a maior incidência no grupo 1 3 anos com mais de $50 \%$ dos casos.

\subsection{Hospitalização}

Na Tabela 4 pode-se observar certa homogeneidade na freqüiência de crianças hospitalizadas nos diversos grupos etários estudados, bem como a média de dias de internação quando se observa aquelas idades em que houve um maior número de pacientes.
Fica a impressão de que não há diferença na gravidade da doença, medida pela internação hospitalar, nas diversas idades estudadas.

Ressalve-se, entretanto, que foram gastos 758 leitos/dias, ou seja, pouco mais de dois anos leito/dia para atender ao sarampo ocorrido na faixa etária de crianças que se encontram entre $2-7$ anos em $1 / 5$ da área urbana de São José do Rio Prêto, nestes últimos 7 anos.

\subsection{Diagnóstico}

Pela Tabela 5 podemos ter uma primeira idéia de como o sarampo é encarado em nossas populações pela sua aparente benignidade na evolução normal. Assim é que apenas para $35 \%$ dos casos houve diagnóstico médico, sendo os demais reconhecidos apenas pela experiência leiga; surpreendentemente, nem o farmacêutico foi, por esta razão, muito soli-

\section{TA B E L A 4}

Internacão hospitalar devido ao sarampo Grupo etário: com $2-17$ anos

São José do Rlo Prêto - CSE - 1973

\begin{tabular}{|c|c|c|c|c|}
\hline \multirow{3}{*}{$\begin{array}{l}\text { Grupo } \\
\text { etário }\end{array}$} & \multicolumn{3}{|c|}{ Tiveram sarampo } & \multirow{3}{*}{$\begin{array}{l}\text { Média } \\
\text { (dias) }\end{array}$} \\
\hline & \multirow{2}{*}{ N.o } & \multicolumn{2}{|c|}{ Internadas } & \\
\hline & & $\mathrm{n}$ & $\%$ & \\
\hline $0-1$ & 85 & 10 & 11,1 & 7,9 \\
\hline $1-2$ & 155 & 28 & 18,0 & 9,3 \\
\hline $2-13$ & 140 & 16 & 11,4 & 15,4 \\
\hline $3-14$ & 86 & 5 & 5,8 & 5,2 \\
\hline $4-15$ & 65 & 12 & 18,4 & 8,9 \\
\hline $5-16$ & 33 & 4 & 12,1 & 8.7 \\
\hline $6-17$ & 5 & .1 & 2.0 & 3.0 \\
\hline Total & 569 & 76 & 13,3 & 9,9 \\
\hline
\end{tabular}


BOSKOVITZ, E. P. et al. - Levantamento de características epidemiológicas do sarampo em São José do Rio Prêto (Brasil) - 1973. Rev. Saúde públ., S. Paulo, 8:181-6, 1974.

citado como aquele elemento intermediário entre o leigo e o médico.

Investigações sociológicas neste campo deverão merecer o aprofundamento do estudo em questão.

TABEL 5

Sarampo - Diagnóstico

São José do Rio Prêto - 1973 - Área do CSE

\begin{tabular}{l|c|c}
\hline \multirow{2}{*}{ Diagnóstico } & \multicolumn{2}{|c}{ f } \\
\cline { 2 - 3 } & $\mathrm{n}$ & $\%$ \\
\hline Experiência própría & 255 & 42,9 \\
Familiares & 54 & 9,0 \\
Farmacêutico & 75 & 12,6 \\
Médico & 310 & 35,3 \\
\hline Total & 594 & 99,8 \\
\hline
\end{tabular}

\section{D I S C S S $\AA 0$}

Publicações recentes têm evidenciado a validade do método da entrevista em inquéritos epidemiológicos sobre o sarampo.

Trabalhos na India ${ }^{4}$ e nos EUA ${ }^{2}$, relacionando a informação familiar e os níveis sorológicos de anti-corpos específicos, animaram-nos a fazer um levantamento de características epidemiológicas do sarampo em área urbana, mesmo sem contar com a utilização de métodos mais sofisticados.

Considerando a grande "experiência" leiga no diagnóstico desta afecção e o uso rotineiro da caderneta de vacinação nos serviços de imunização, pode-se conhecer com relativa precisão a incidência do sarampo nos grupos etários inferiores e a possível ação preventiva da vacina. Os resultados observados, com o evidente des- locamento das maiores incidências para as menores idades, deverão ser melhor acompanhados nos anos seguintes. Haveria uma real diminuição no grupo dos suscetíveis mais velhos pela imunização ativa natural, sendo o pequeno número de vacinados precocemente a razão do maior número de crianças com sarampo, nos grupos $0|-1,1|-2$ anos de idade? Seria já o efeito profilático da vacina? Não acreditamos nesta alternativa pois, apesar da vacinação com virus atenuado já ter-se iniciado nos EUA em $1963^{1}$, em nosso meio, somente a partir de 1969-70 é que passou a ser realmente difundido $o$ seu uso.

Quanto à hospitalização é de se estranhar que não tenha sido mais elevada nos grupos etários menores, onde se prevê, em geral, maiores complicações clínicas como se observou na Checoslováquia em $5 \%$ dos casos ${ }^{6}$.

Lamentando não ter sido possível saber o diagnóstico hospitalar (falta do registro de dados), fica-se com a impressão de que a internação tenha ocorrido simplesmente por decorrência da própria doença, oportunidade do atendimento, vaga hospitalar, etc.

Saliente-se, tão somente o elevado custo e a demanda exigida por uma doença, cujos métodos preventivos eficazes jả eram por demais conhecidos no período estudado.

\section{CONCLUSOES}

Com recursos relativamente limitados pode-se desenvolver um levantamento epidemiológico do sarampo com caráter didático e de investigação.

Pode-se evidenciar o valor profilático da vacina atenuada contra o sarampo em nosso meio.

Observou-se maior incidência do sarampo no grupo etário $1-3$ anos em área urbana, quando estudada a cohorte de nascidos entre 1966-71 (grupo $2-7$ anos atuais). 
BOSKOVITZ, E. P. et al. - Levantamento de caracteristicas epidemiológicas do sarampo em São José do Rio Prêto (Brasil) - 1973. Rev. Saúde públ., S. Paulo, 8:181-6, 1974.

A freqüência da hospitalização pelo sarampo não variou significativamente no período e idade estudados. A média de dias de internação foi de 9,9 acarretando uma demanda de 758 leitos/dia, nos últimos 5 anos, para a amostra estudada em São José do Rio Prêto.

Apenas $35 \%$ dos casos de sarampo foram diagnosticados por médico; os demais por leigos, pontifiacndo a "experiência própria" em $42,9 \%$ dos casos estudados, revelando a conduta de população urbana frente a uma das doenças da infância altamente prevalentes em nosso meio.

\section{A GRADECIMENTOS}

Ao doutorando Irineu Luis Maia, preceptor do Departamento de Medicina Preventiva e aos alunos da $2 .^{\mathrm{a}}$ série médica da Faculdade Regional de Medicina de São José do Rio Prêto (1973) que muito colaboraram para a consecussão deste trabalho.

Boskovitz, E. P. et al. - [Appraisal of the epidemiological characteristics of measles in São José do Rio Prêto (Brazil) - 1973.1 Rev. Saúde públ., S. Paulo, 8:181-6, 1974

SUMMARY: In this country measles is considered a very important infection from the sanitary point of view. Its incidence was studied in a cohort of 5 years (between 2 and 7 years old at present) in the urban area of São José do Rio Prêto. The progressive increase of incidence in the lower age patients, was showed. The prophylactie value of specific vaccination $(96 \%)$ and the hospital demand due to measles ( 758 beds/day) were evaluated by means of the most accessible mean of investigation in this area - i.e., personnal interviews.

UNITERMos: Measles*; Epidemiology*; Morbidity*; Vaccine, prophilaxis; Hospital Demend.

\section{REFERENCIAS BIBLIOGRAFICAS}

1. AXNICH, M. W. et al. - Beneficios de la inmunización contra el sarampión. Bol. Ofic. sanit. panamer., 68:201-9, 1970.

2 CHERRY, J. D. et al. - Urban Measles in the vaccine era: a clinical, epidemiologic and serologic study. $J$. Pediat., 81:217-30, 1972.

3. LINNEMANN, C. C. - Measles vaccine: immunity, reinfection and revaccination. Amer. J. Epidem., 97:365-71, 1973.

4. MENEVATI, A. M. D. \& NASEEME, A. M. - Measles surveys, Bull. Wld. Hith. Org., 47:543-684, 1972.
5. PUFFER, R. R. et al. - Investigación interamericana de mortalidad en la niñez: informe provisional, 1968/1969. Washington, D.C. Organizacion Panamericana de la Salud, 1971.

6. VIGILANCIA epidemiológica del sarampión. Bol. Ofic. sanit. panamer., 71: 436-9, 1971. 